\title{
Low-Energy Predictions of Lopsided Family Charges
}

\author{
J. Sato \\ Research Center for Higher Education, Kyushu University, Ropponmatsu, Chuo-ku, Fukuoka, 810-8560, Japan \\ and \\ T. Yanagida \\ Department of Physics and RESCEU, University of Tokyo, Hongo, Bunkyo-ku, Tokyo 113-0033, Japan
}

November 28, 2018

\begin{abstract}
We consider the Froggatt-Nielsen (FN) mechanism reproducing the observed mass hierarchies and mixing angles for quarks and leptons. The large $\nu_{\mu}-\nu_{\tau}$ mixing suggested from recent Superkamiokande experiments on the atmospheric neutrinos implies lopsided FN U(1) charges for the lepton doublets. There are two possible charge assignments to generate the large $\nu_{\mu}-\nu_{\tau}$ mixing. We point out that the two models with different charge assignments have distinct low-energy predictions and hence they are distinguishable in future neutrino experiments on such as $\mathrm{CP}$ violation and $2 \beta 0 \nu$ decay.
\end{abstract}

\section{Introduction}

The observed quark-lepton mass spectra and mixing angles may provide us with important information on a more fundamental theory beyond the standard model. There have been proposed many mechanisms and symmetries to account for the observed mass spectra and mixings for quarks and leptons. Among them we consider that the Froggatt-Nielsen(FN) mechanism 1] using a broken U(1) family symmetry is the simplest and the most promising candidate. In this scheme the masses and mixings for quarks are well understood by choosing properly the FN U(1) charges for each quarks.

The large $\nu_{\mu}-\nu_{\tau}$ mixing observed by Superkamiokande experiments on atmospheric neutrinos 2$]$ has led us to propose lopsided $\mathrm{U}(1)$ charges to the left-handed lepton doublets $l_{i}(i=1-3) 3$. 4 . Namely, we propose that the lepton doublets $l_{2}$ and $l_{3}$ of the second and third families have the same $\mathrm{U}(1)$ charges $A$ and the lepton doublet $l_{1}$ of the first family has the $\mathrm{U}(1)$ charge $A+1[3$, while the right-handed charged leptons $\bar{e}_{i}(i=1-3)$ have the $\mathrm{U}(1)$ charges, 2,1,0, respectively(model I). This lopsided charge assignment for $l_{i}$ is a crucial point to produce the large mixing between $\nu_{\mu}$ and $\nu_{\tau}$. However, the above choice of $\mathrm{U}(1)$ charge is not unique to reproduce the large $\nu_{\mu}$ and $\nu_{\tau}$ mixing. That is, there is another charge assignment that all lepton doublets $l_{i}$ have the same charge $A$ (model II) [5], where the right-handed charged leptons $\bar{e}_{i}$ have the FN U(1) charges 3,1,0.

In this letter we show that the above two charge choices lead to distinct low-energy predictions and hence the two models can be testable in future neutrino experiments on such as CP violation in neutrino oscillation and $2 \beta 0 \nu$ decay. We show the FN U(1) charges $Q_{l_{i}}$ for $l_{i}$. in Table 1 . We assume that the $\mathrm{FN} \mathrm{U}(1)$ charge for the Higgs doublet $H$ is zero.

\begin{tabular}{c|c|c} 
& $\mathrm{I}$ & $\mathrm{II}$ \\
\hline$Q_{l_{3}}$ & $A$ & $A$ \\
$Q_{l_{2}}$ & $A$ & $A$ \\
$Q_{l_{1}}$ & $A+1$ & $A$
\end{tabular}

Table 1: U(1) charges for lepton doublets 
In the FN mechanism the $\mathrm{U}(1)$ symmetry is explicitly broken by the vacuum expectation value of $\phi,\langle\phi\rangle$, where the $\mathrm{U}(1)$ charge for $\phi$ is -1 . Then all Yukawa couplings are given by the following form:

$$
\begin{aligned}
L & =h_{i j} \psi_{i} \psi_{j} H\left(\text { or } H^{*}\right)\left(\frac{<\phi>}{M_{*}}\right)^{Q_{\psi_{i}}+Q_{\psi_{j}}}, \\
& \equiv h_{i j} \psi_{i} \psi_{j} H\left(\text { or } H^{*}\right) \epsilon^{Q_{\psi_{i}}+Q_{\psi_{j}}}
\end{aligned}
$$

where $h_{i j}$ is a constant with its norm of $\mathrm{O}(1), Q_{\psi_{i}}$ is the FN charge for the filed $\psi_{i}, M_{*}$ the gravitational scale $M_{*} \simeq 2.4 \times 10^{18} \mathrm{GeV}$ and $\epsilon \equiv \frac{\langle\phi\rangle}{M_{*}}$.

Then, the neutrino Dirac mass matrix in the model I is given by 1

$$
\begin{aligned}
M_{D} & =\epsilon^{A} m_{0}\left(\begin{array}{ccc}
h_{11} \epsilon^{c+1} & h_{12} \epsilon^{b+1} & h_{13} \epsilon^{a+1} \\
h_{21} \epsilon^{c} & h_{22} \epsilon^{b} & h_{23} \epsilon^{a} \\
h_{31} \epsilon^{c} & h_{32} \epsilon^{b} & h_{33} \epsilon^{a}
\end{array}\right) \\
& =\epsilon^{A} m_{0}\left(\begin{array}{ccc}
h_{11} \epsilon & h_{12} \epsilon & h_{13} \epsilon \\
h_{21} & h_{22} & h_{23} \\
h_{31} & h_{32} & h_{33}
\end{array}\right)\left(\begin{array}{ccc}
\epsilon^{c} & 0 & 0 \\
0 & \epsilon^{b} & 0 \\
0 & 0 & \epsilon^{a}
\end{array}\right)
\end{aligned}
$$

and that in the model II takes the following form

$$
\begin{aligned}
M_{D} & =\epsilon^{A} m_{0}\left(\begin{array}{lll}
h_{11} \epsilon^{c} & h_{12} \epsilon^{b} & h_{13} \epsilon^{a} \\
h_{21} \epsilon^{c} & h_{22} \epsilon^{b} & h_{23} \epsilon^{a} \\
h_{31} \epsilon^{c} & h_{32} \epsilon^{b} & h_{33} \epsilon^{a}
\end{array}\right) \\
& =\epsilon^{A} m_{0}\left(\begin{array}{lll}
h_{11} & h_{12} & h_{13} \\
h_{21} & h_{22} & h_{23} \\
h_{31} & h_{32} & h_{33}
\end{array}\right)\left(\begin{array}{ccc}
\epsilon^{c} & 0 & 0 \\
0 & \epsilon^{b} & 0 \\
0 & 0 & \epsilon^{a}
\end{array}\right)
\end{aligned}
$$

The Majorana mass term for right-handed neutrinos $\nu_{R}$ takes a similar form

$$
\begin{aligned}
M_{\nu_{R}} & =M_{R}\left(\begin{array}{ccc}
m_{11} \epsilon^{2 c} & m_{12} \epsilon^{c+b} & m_{13} \epsilon^{c+a} \\
m_{12} \epsilon^{b+c} & m_{22} \epsilon^{2 b} & m_{23} \epsilon^{b+a} \\
m_{13} \epsilon^{a+c} & m_{23} \epsilon^{a+b} & m_{33} \epsilon^{2 a}
\end{array}\right) \\
& =M_{R}\left(\begin{array}{ccc}
\epsilon^{c} & 0 & 0 \\
0 & \epsilon^{b} & 0 \\
0 & 0 & \epsilon^{a}
\end{array}\right)\left(\begin{array}{ccc}
m_{11} & m_{12} & m_{13} \\
m_{12} & m_{22} & m_{23} \\
m_{13} & m_{23} & m_{33}
\end{array}\right)\left(\begin{array}{ccc}
\epsilon^{c} & 0 & 0 \\
0 & \epsilon^{b} & 0 \\
0 & 0 & \epsilon^{a}
\end{array}\right),
\end{aligned}
$$

where we assume that $Q_{\nu_{R}}$ for right-handed neutrinos are $a, b, c$, and $m_{0}$ and $M_{R}$ represent weak scale and right-handed neutrino mass scale, respectively. Here $m_{i j}$ is a constant with its norm of $\mathrm{O}(1)$ like the coupling $h_{i j}$. For numerical convenience we take a basis where the charged leptons are diagonalized, throughout this letter except when we determine the $\epsilon$ parameter using the charged lepton mass matrix (9).

From eq.(2), (3) and (4) we have the following Majorana mass term for left-handed neutrinos $\nu_{L}[6]$ in the model I

$$
\begin{aligned}
& M_{\nu_{L}} \\
= & \frac{\epsilon^{2 A} m_{0}^{2}}{M_{R}}\left(\begin{array}{ccc}
h_{11} \epsilon & h_{12} \epsilon & h_{13} \epsilon \\
h_{21} & h_{22} & h_{23} \\
h_{31} & h_{32} & h_{33}
\end{array}\right)\left(\begin{array}{lll}
m_{11} & m_{12} & m_{13} \\
m_{12} & m_{22} & m_{23} \\
m_{13} & m_{23} & m_{33}
\end{array}\right)^{-1}\left(\begin{array}{ccc}
h_{11} \epsilon & h_{12} \epsilon & h_{13} \epsilon \\
h_{21} & h_{22} & h_{23} \\
h_{31} & h_{32} & h_{33}
\end{array}\right)^{T} \\
\sim & \left(\begin{array}{ccc}
\epsilon^{2} & \epsilon & \epsilon \\
\epsilon & 1 & 1 \\
\epsilon & 1 & 1
\end{array}\right)
\end{aligned}
$$

\footnotetext{
${ }^{1}$ The Dirac mass matrix $M_{D}$ is defined as $L=\left(\nu_{L}\right)_{i} M_{D i j} \bar{\nu}_{R}+$ h.c.
} 
and in the model II

$$
\begin{aligned}
& M_{\nu_{L}} \\
= & \frac{\epsilon^{2 A} m_{0}^{2}}{M_{R}}\left(\begin{array}{lll}
h_{11} & h_{12} & h_{13} \\
h_{21} & h_{22} & h_{23} \\
h_{31} & h_{32} & h_{33}
\end{array}\right)\left(\begin{array}{lll}
m_{11} & m_{12} & m_{13} \\
m_{12} & m_{22} & m_{23} \\
m_{13} & m_{23} & m_{33}
\end{array}\right)^{-1}\left(\begin{array}{lll}
h_{11} & h_{12} & h_{13} \\
h_{21} & h_{22} & h_{23} \\
h_{31} & h_{32} & h_{33}
\end{array}\right)^{T} \\
\sim & \left(\begin{array}{lll}
1 & 1 & 1 \\
1 & 1 & 1 \\
1 & 1 & 1
\end{array}\right)
\end{aligned}
$$

Note that the FN charges of $\nu_{R}$ 's are irrelevant to the above $M_{\nu_{L}}$ 's. Therefore, we take $a=b=c=0$ in the present analysis.

We randomly generate the coefficients $h_{i j}$ and $m_{i j}$ such that their magnitudes be between 0.8 and 1.2 and their complex phases be distributed from 0 to $2 \pi$, and calculate the lepton mixing angles and the mass square differences for each generated parameters. We require that those mixings and the mass square differences satisfy the conservative constraints from the current experiments [7]:

A. $\left|U_{e 3}\right|<0.15$ to satisfy the CHOOZ limit. 8

B. $4\left|U_{\mu 3}\right|^{2}\left(1-\left|U_{\mu 3}\right|^{2}\right)>0.5$ to have the large mixing for atmospheric neutrino oscillation. 2

C. To satisfy the constraint from solar neutrino deficit, one of the following two conditions is required to be satisfied: 9

(a) For the small angle solution, $10^{-4}<r<10^{-2}$ and $10^{-4}<\tan ^{2} \theta<5 \times 10^{-3}$.

(b) for the large angle solution, $r<0.1$ and $10^{-1}<\tan ^{2} \theta<10$.

Here $\tan ^{2} \theta \equiv\left|U_{e 2} / U_{e 1}\right|^{2}$ and $r$ is the ratio between the smallest mass square difference and the second smallest one, i.e $r \equiv \delta m_{\text {solar }}^{2} / \delta m_{\text {atm }}^{2}$.

Notice that the criterion $\mathrm{A}$ is automatically satisfied in the model I, while $\left|U_{e 3}\right| \sim \mathrm{O}(1)$ generally in the model II.

To calculate the Majorana masses for left-handed neutrinos in the model I, we need to fix the value of $\epsilon$. To find how small value we should take for $\epsilon$, we calculate the charged lepton masses with $Q_{e_{i}}=(0,1,2)$,

$$
M_{l} \propto\left(\begin{array}{ccc}
l_{11} \epsilon^{3} & l_{12} \epsilon^{2} & l_{13} \epsilon^{1} \\
l_{21} \epsilon^{2} & l_{22} \epsilon^{1} & l_{23} \epsilon^{0} \\
l_{31} \epsilon^{2} & l_{32} \epsilon^{1} & l_{33} \epsilon^{0}
\end{array}\right),
$$

where $l_{i j}$ 's are randomly generated coefficients in the same way as $h_{i j}$ and $m_{i j}$. To see how easily we can have a solution for a given set of coefficients, we randomly generate 1000000 sets of the coefficients and find how many sets can satisfy the following cuts.

$$
\begin{aligned}
14 & <\frac{m_{\tau}}{m_{\mu}}<20 \\
180 & <\frac{m_{\mu}}{m_{e}}<240
\end{aligned}
$$

The number of sets satisfying these cuts depends on the value of $\epsilon$. From the dependence on $\epsilon$ of it in fig.(1), we can find that $\epsilon$ is likely in the range between 0.05 and 0.13 We find that the number of sets in the model II satisfying the same cuts (eq, 10) ) is almost the same as that in the model I if the right-handed charged leptons $\bar{e}_{i}$ have the FN U(1) charges 3,1,0.

From now on we show the results. We generate one million sets of coefficients for each $\epsilon=$ $(0.05,0.06,0.07,0.08,0.09,0.1)$ in the model $\mathrm{I}$ and in the model II. Note that the parameter $\epsilon$ is irrelevant to the neutrino mass matrix (see eq.(7)) in the model II. First we see how many sets can remain after the constraints (A,B and C) are imposed. It is summarized in table 2] where we list the number of sets separating the cases of small and large mixing solar neutrino solutions.

\footnotetext{
${ }^{2}$ In this letter we take the range between 0.8 and 1.2 for the norm $\left|h_{i j}\right|$ and $\left|m_{i j}\right|$. However, the results do not change much even if one takes a wider range of the norms, say 0.5-1.5.

${ }^{3}$ The absolute values of the vertical axis depends on how tightly we select samples, so only the shape of the graph should be considered.
} 


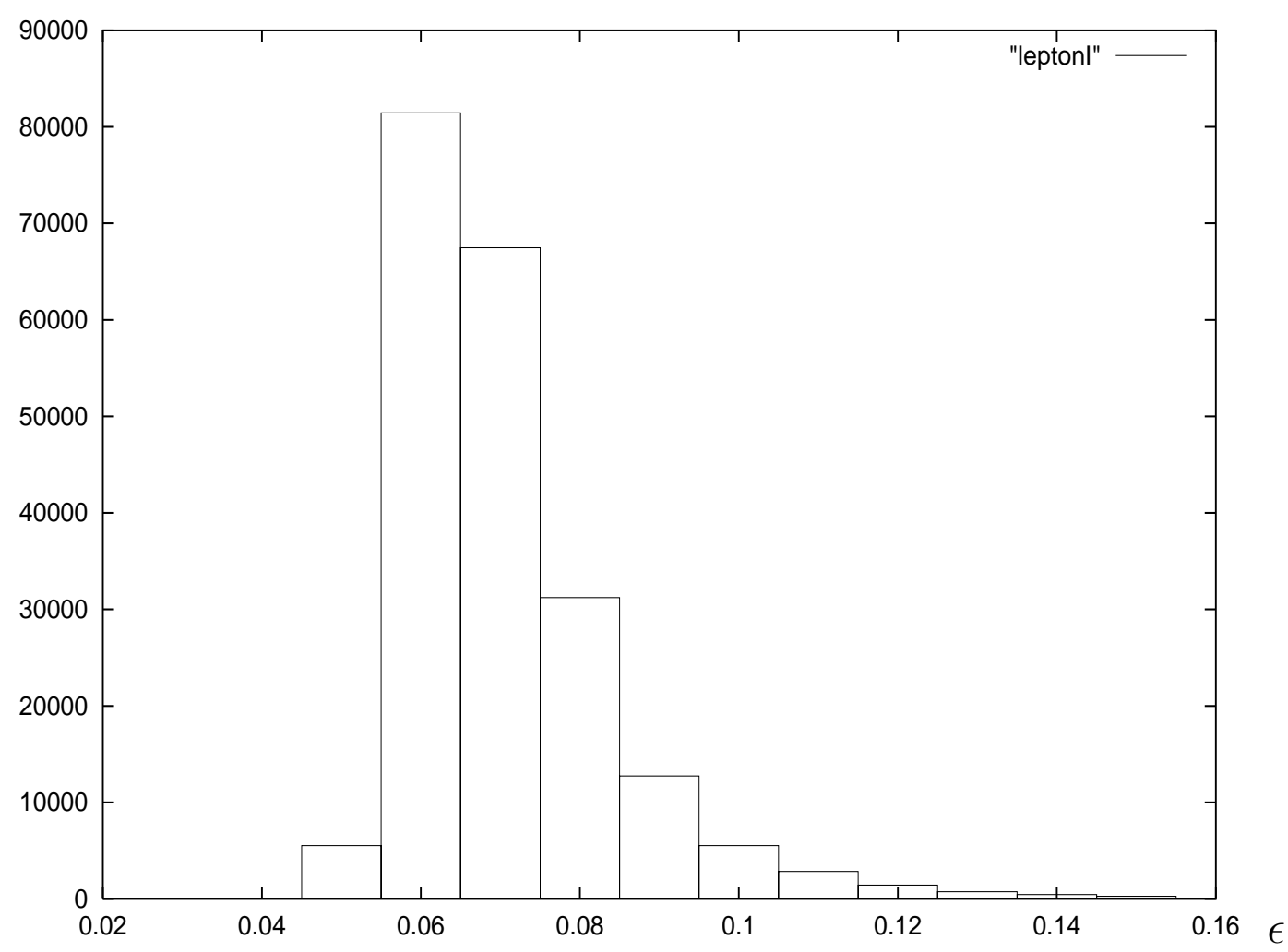

Figure 1: Dependence on $\epsilon$ of how easily we can get solutions for lepton masses. The shape of the dependence does not change with the tightness of the selection.

In the model I, we have about $10 \%$ sets of coefficients as solutions independently of $\epsilon$. This was first pointed out in ref. 10. On the contrary, in the model II about $1 \%$ sets can satisfy the criterion 4 This lower probability (1\%) comes mainly from the constraint $\left|U_{e 3}\right|<0.15$. We have also small mixing angle solutions in the model I, whose physical reason will be discussed later. In fig. 2 we show the relation between the solar angle $\tan ^{2} \theta \equiv\left|U_{e 2} / U_{e 1}\right|^{2}$ and the ratio of the mass square differences $r$.

In the model II $r$ and $\tan ^{2} \theta$ distribute almost uniformly while in the model I, there is a disfavored region at right top end for the large angle solutions. This disfavored region can be understood in the following way. First we note that the Majorana mass matrix takes the form of eq.(6). By diagonalizing the dominant 2 by 2 part, we will have the following mass matrix,

$$
\left(\begin{array}{ccc}
\epsilon^{2} & \epsilon & \epsilon \\
\epsilon & \delta & 0 \\
\epsilon & 0 & 1
\end{array}\right),
$$

in which $\delta^{2}$ corresponds to $r$ approximately. At the same time

$$
\tan \theta \simeq \epsilon / \delta \text { for } \epsilon<\delta \text {. }
$$

Thus

$$
r \times \tan ^{2} \theta \simeq \epsilon^{2}
$$

and hence $\tan ^{2} \theta>1$ can be hardly obtained for $r \simeq 0.1$ and $\epsilon \simeq 0.1$.

\footnotetext{
${ }^{4}$ Note that the model II is essentially different from the idea of anarchy 11, where the norms of all Yukawa couplings vary from 0 to 1 . Since they can take very small values $\sim 0$, the probability realizing small $U_{e 3}$ increases.
} 


\begin{tabular}{l|c|c} 
& small mixing & large mixing \\
\hline \hline $\mathrm{I}(\epsilon=0.05)$ & 81818 & 20025 \\
\hline $\mathrm{I}(\epsilon=0.06)$ & 64353 & 28837 \\
\hline $\mathrm{I}(\epsilon=0.07)$ & 51867 & 38638 \\
\hline $\mathrm{I}(\epsilon=0.08)$ & 42436 & 49616 \\
\hline $\mathrm{I}(\epsilon=0.09)$ & 34714 & 61220 \\
\hline $\mathrm{I}(\epsilon=0.1)$ & 29330 & 72372 \\
\hline \hline $\mathrm{II}$ & 6 & 9703
\end{tabular}

Table 2: Sample of how many sets can satisfy the criterion. Here, "small(large) mixing" implies that the set satisfies the criterion for the small(large) mixing angle solution to the solar neutrino problem.

The above arguement also shows the reason why we obtain the large mixing angle solution for solar neutrino. However it is not complete. Due to the uncertainties in the right-handed neutrino Majorana mass matrix, the 33 element in eq. (111) can be rather large and hence there is a possibility to make $r$ sufficiently small keeping $\delta \sim \mathrm{O}(1)$. In this case the small mixing angle solution may be obtained. This possibility was not found in [10, since the author of ref [10] considered only the effective operator,

$$
L=\frac{H^{2}}{M_{R}} \kappa_{i j} \nu_{L i} \nu_{L j}
$$

Next we see the distribution of $U_{e 3}$ which is one of the most important parameters in the next generation neutrino oscillation experiments. In fig. 3] we show the distributions of $U_{e 3}$ for both models which satisfy our criterion (A,B and C). From eq.(6), $U_{e 3}$ in the model I is expected to be proportional to $\epsilon$. Indeed this scaling can be seen numerically. On the contrary, since there is no symmetry which distinguishes the generation in the model II, $U_{e 3}$ is likely to be large and indeed this is seen in fig. [3 In both models $U_{e 3}$ is expected to be large enough to be observed in future oscillation experiments like neutrino factory [12].

Next we consider how large CP violation can be seen in neutrino oscillation experiments. The magnitude of CP violation is characterized by the Jarlskog parameter 13] with the Paticle Data Group notation for mixing matrix [14]

$$
\begin{aligned}
J & \equiv\left|\operatorname{Im}\left(U_{\alpha i}^{*} U_{\beta j}^{*} U_{\alpha j} U_{\beta i}\right)\right| \\
& =\left|\operatorname{Im}\left(U_{e 3}^{*} U_{\mu 2}^{*} U_{e 2} U_{\mu 3}\right)\right| \\
& =\frac{1}{4}\left|\sin \theta_{13} \cos ^{2} \theta_{13} \sin 2 \theta_{23} \sin 2 \theta_{12} \sin \delta\right|
\end{aligned}
$$

Here $\sin \theta_{13}$ is $U_{e 3}, \theta_{23}$ and $\theta_{12}$ almost correspond to $\theta_{\text {atm }}$ and $\theta_{\text {solar }}$, respectively and $\delta$ is the CP violating phase. The distributions of $|J|$ are shown in fig 4 To draw the graph for the model I, we use only samples which reproduce the large angle solution for solar neutrino, since samples with the small angle solution make $J$ much smaller which may not be observable.

The dependence on $\epsilon$ of $J$ in the model I is $J \sim \epsilon$ since $U_{e 3}$ is proportional to $\epsilon$ as we see in fig[3] while the other angles including $\mathrm{CP}$ violating phase are of $\mathrm{O}(1)$. That is,

$$
\begin{aligned}
J & \sim \underset{\text { prefactor }}{0.25} \times \begin{array}{c}
U_{e 3} \\
\end{array} \\
& \simeq 0.1 \epsilon .
\end{aligned}
$$

On the other hand, $J$ in the model II is estimated to be

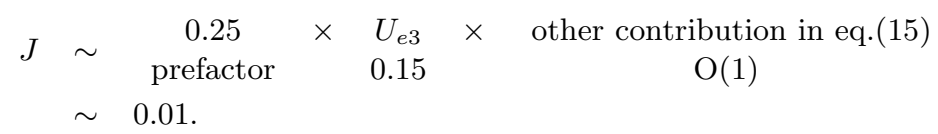

and hence slightly larger than that in the model I. 
In the realistic neutrino oscillation experiment, the measurable quantity for CP violation is not $J$ itself but 15

$$
\tilde{J} \equiv\left|J \times \frac{\delta m_{\text {solar }}^{2}}{\delta m_{\text {atm }}^{2}}\right| .
$$

In fig 5 we plot the distribution of $|\tilde{J}|$. The dependence on $\epsilon$ of $\tilde{J}$ in the model I is rather complicated. Due to eq. (13),

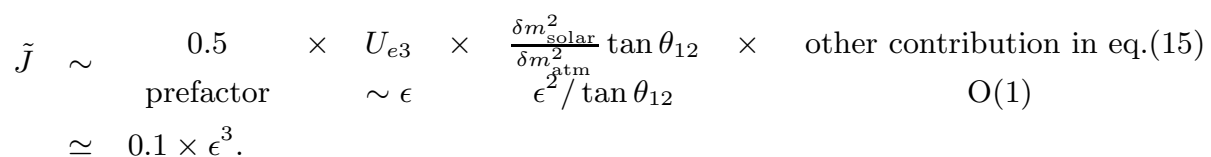

On the contrary $\tilde{J}$ in the model II is estimated easily as

$$
J \times \frac{\delta m_{\text {solar }}^{2}}{\delta m_{\text {atm }}^{2}} \sim 0.01 \times(0.1-0.01) \sim(0.001-0.0001) .
$$

Thus, there is a possibility to measure CP violation in the next generation neutrino oscillation experiments for the model II while there seems to be little hope to see CP violation in the near future experiments 16 for the model I.

Finally, we calculate $m_{e e} \equiv\left|\sum_{i} U_{e i}^{2} m_{i}\right|$ which is a crucial parameter to determine the $2 \beta 0 \nu$ decay rate. In fig [6] we show the distributions of $m_{e e}$ in the both models. In the figure, we have assumed $\delta m_{\mathrm{atm}}^{2}=3 \times 10^{-3} \mathrm{eV}^{2}$. The dependence on $\epsilon$ of $m_{e e}$ in the model $\mathrm{I}$ is very simple. Due to eq.(6),

$$
\begin{aligned}
m_{e e} & \sim \sqrt{3 \times 10^{-3}} \epsilon^{2} \\
& \simeq 0.05 \times \epsilon^{2} \mathrm{eV}
\end{aligned}
$$

On the contrary $m_{e e}$ in the model II is naively expected to be $\sqrt{3 \times 10^{-3}} \sim 0.05 \mathrm{eV}$. However the samples which satisfy our criterion prefer values lower by almost one order of magnitude.

Again, there is a possibility to find the $2 \beta 0 \nu$ decay in the next generation experiments for the model II while there is little hope to see it in the near future experiments for the model I. 17.

In conclusion, we summarize the results. There are two kinds of FN U(1) charges which realize lopsided structure for the lepton doublets mass matrices, i.e the model I (001) and the model II(000). These two sets of charge assignments have very different feature from each other and hence it is testable in the near future experiments which type is likely the case.

We have considered first the solar neutrino oscillation. In the model II we hardly get a small angle solution to the solar neutrino problem, so if the solar neutrino deficit is explained by the small angle solution, then the model II will be rejected. In the model I there is a disfavored region, which is explained by eq. (13), and hence if it explains the solar neutrino deficit then the model I will be disfavored.

Next we have discussed the distribution of $U_{e 3}$. Unfortunately as is seen in fig 3 it is very difficult to distinguish the model I from the model II by this angle.

Then we have studied CP violation in the lepton sector. As is shown in fig's 4 and 5 it seems difficult to see $\mathrm{CP}$ violation in the next generation neutrino oscillation experiments in the model I, while there is a possibility to observe it in the model II. Therefore, if we detect the CP violation in the lepton sector then the model II will be favored.

Finally, we have examined how large $m_{e e} \equiv \sum_{i} U_{e i}^{2} m_{i}$ can be, which is a key element for $2 \beta 0 \nu$ decay. The distribution of it in the model I shows the expected shape from eq. (21) as is seen in fig 6 while in the model II its magnitude is smaller almost by one order of magnitude than that naively expected. However, $m_{e e}$ in the model II lies in the range accessible in the near future experiments while it will be harder to see $2 \beta 0 \nu$ decay in the model I.

\section{Acknowledgments}

The authors are grateful to N. Haba and H. Murayama for useful discussions. The work of J. S is supported in part by a Grant-in-Aid for Scientific Research of the Ministry of Education, Science and Culture, \#12047221, \#12740157. The work of T.Y is supported in part by the Grant-in-Aid, Priority Area "Supersymmetry and Unified Theory of Elementary Particles"(\#707). 


\section{References}

[1] C.D. Froggatt and H.B. Nielsen, Nucl. Phys. B147 (1979) 277.

[2] Y. Fukuda et al. [Superkamiokande Collaboration], Phys. Lett. B433 (1998) 9; Phys. Lett. B436 (1998) 33; Phys. Rev. Lett. 81 (1998) 1562.

[3] J. Sato and T. Yanagida, Phys. Lett. B430 (1998) 127; Nucl. Phys. B (Proc. Suppl.) 77 (1999) 293.

W. Buchmuller and T. Yanagida, Phys. Lett. B445 (1999) 399.

[4] C. H. Albright, K.S. Babu and S.M. Barr , Phys. Rev. Lett. 81 (1998) 1167.

N. Irges, S. Lavignac and P. Ramond, Phys. Rev. D58 (1998) 035003.

[5] J. Hisano , K. Kurosawa and Y. Nomura, Nucl. Phys. B584 (2000) 3.

[6] T. Yanagida, in Proc. Workshop on the unified theory and the baryon number in the universe, (Tsukuba, 1979), eds. O. Sawada and S. Sugamoto, Report KEK-79-18 (1979);

M. Gell-Mann, P. Ramond and R. Slansky, in "Supergravity" (North-Holland, Amsterdam, 1979) eds. D.Z. Freedman and P. van Nieuwenhuizen.

[7] G.L. Fogli, E. Lisi, A. Marrone and G. Scioscia Phys. Rev. D59 (1999) 033001

M.C. Gonzalez-Garcia and C. Pea-Garay, hep-ph/0009041

[8] M. Apollonio et al., Phys. Lett. B466 (1999) 415.

[9] Super-Kamiokande Collaboration, Y. Fukuda et al., Phys. Rev. Lett. 81 (1998) 1158 ; Erratum 81 (1998) 4279 ; 82 (1999) 1810; 82 (1999) 2430 ; Y. Suzuki, Nucl. Phys. B (Proc. Suppl.) 77 (1999) 35.

[10] F. Vissani, JHEP 9811 (1998) 025.

[11] L. Hall, H. Murayama and N. Weiner, Phys. Rev. Lett. 84 (2000) 2572.

[12] S. Geer, Phys. Rev. D57 (1998) 6989, Erratum-ibid D59 (1999) 039903.

[13] C. Jarlskog, Phys. Rev. Lett. 55 (1985) 1039.

[14] Particle Data Group Eur. Phys. J. C15 (2000) 1.

[15] J. Arafune, M. Koike and J. Sato, Phys. Rev. D56 (1997) 3093, Erratum-ibid. D60 (1999) 119905.

[16] J Sato, hep-ph/0008056

B. Richter, hep-ph/0008222

A. Cervera, A. Donini, M.B. Gavela, J.J. Gomez Cadenas, P. Hernandez, O. Mena and S. Rigolin, Nucl. Phys. B579 (2000)17.

[17] J. Hellmig and H. V. Klapdor-Kleingrothaus, Z. Phys. A359 (1997) 351.

Klapdor-Kleingrothaus and M. Hirsch, Z. Phys. A359 (1997) 361. 
$r=\frac{\delta m_{\text {solar }}^{2}}{\delta m_{\text {atm }}^{2}}$

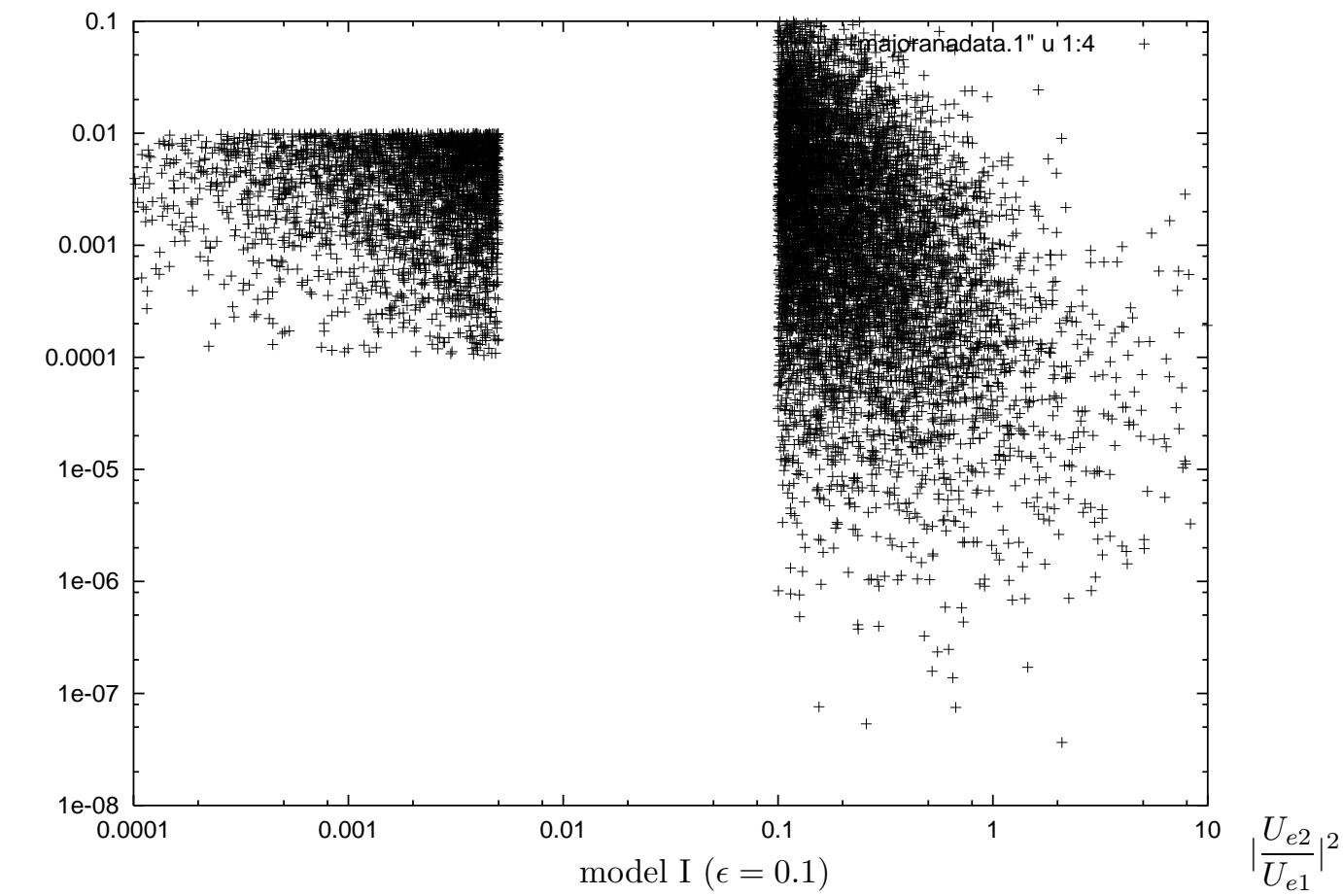

$r=\frac{\delta m_{\text {solar }}^{2}}{\delta m_{\text {atm }}^{2}}$

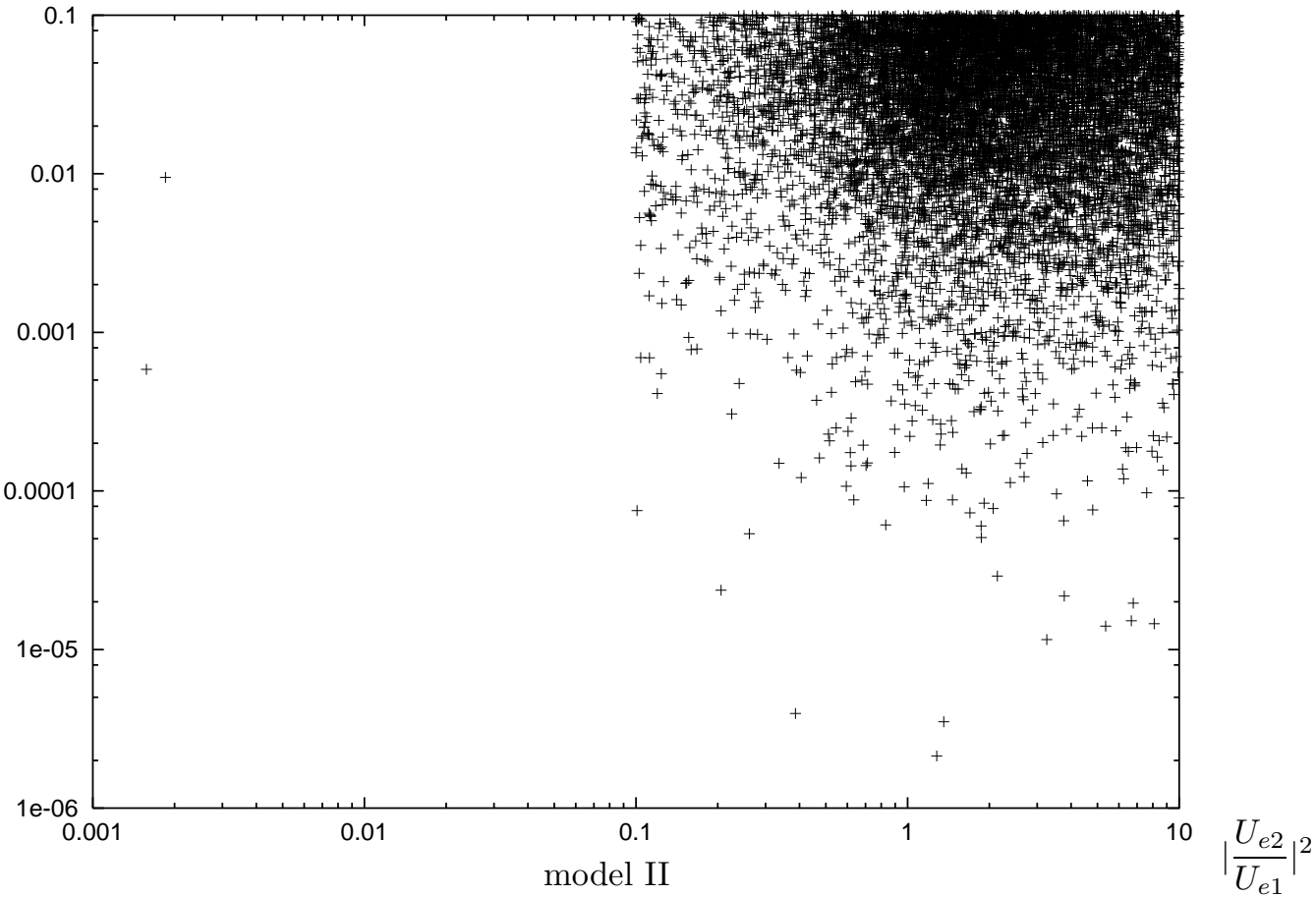

Figure 2: Relations between the mixing angles and mass ratio. The shape of the dependence does not change much with the tightness of the selection. 

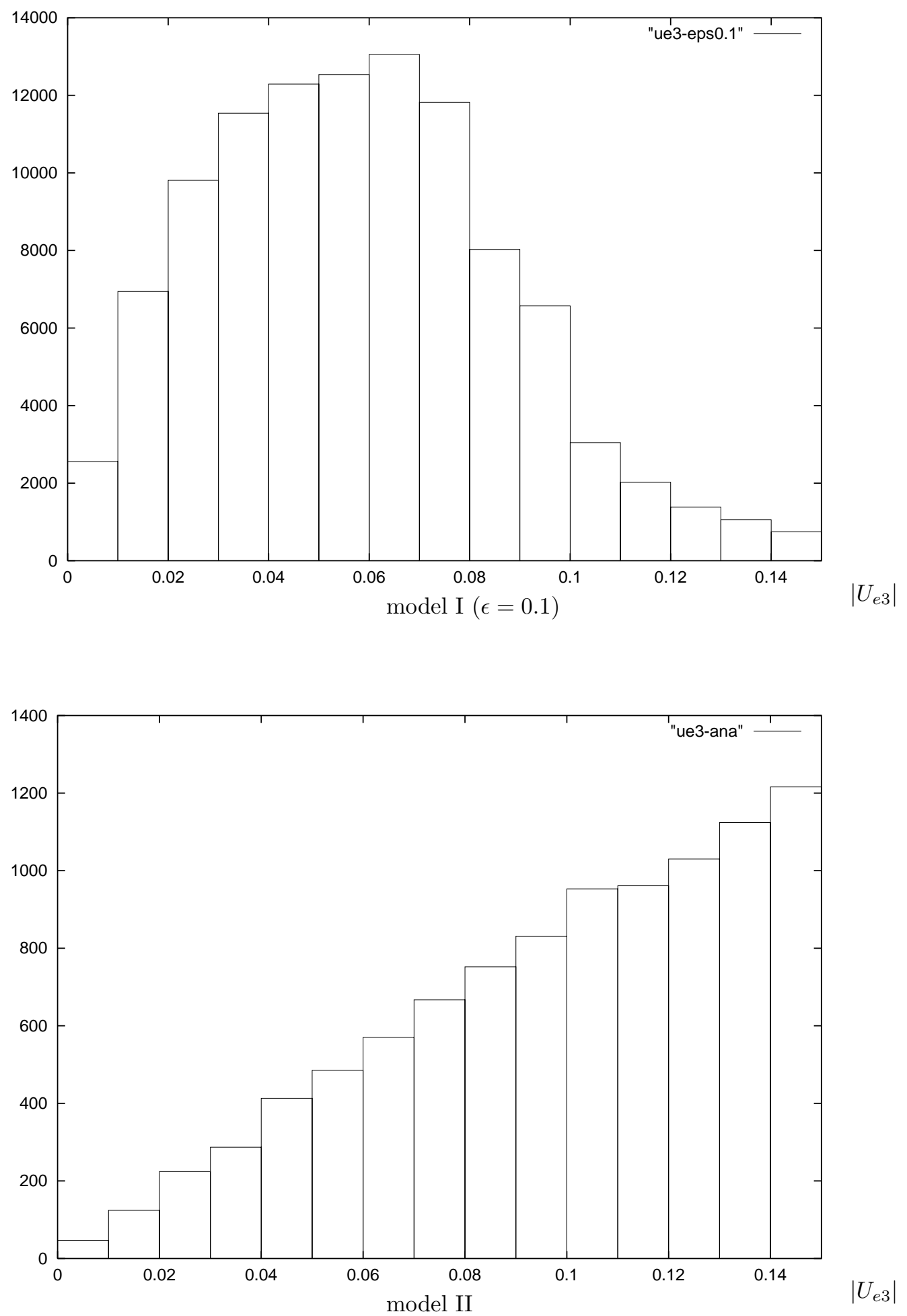

Figure 3: Distributions of $\left|U_{e 3}\right|$. 

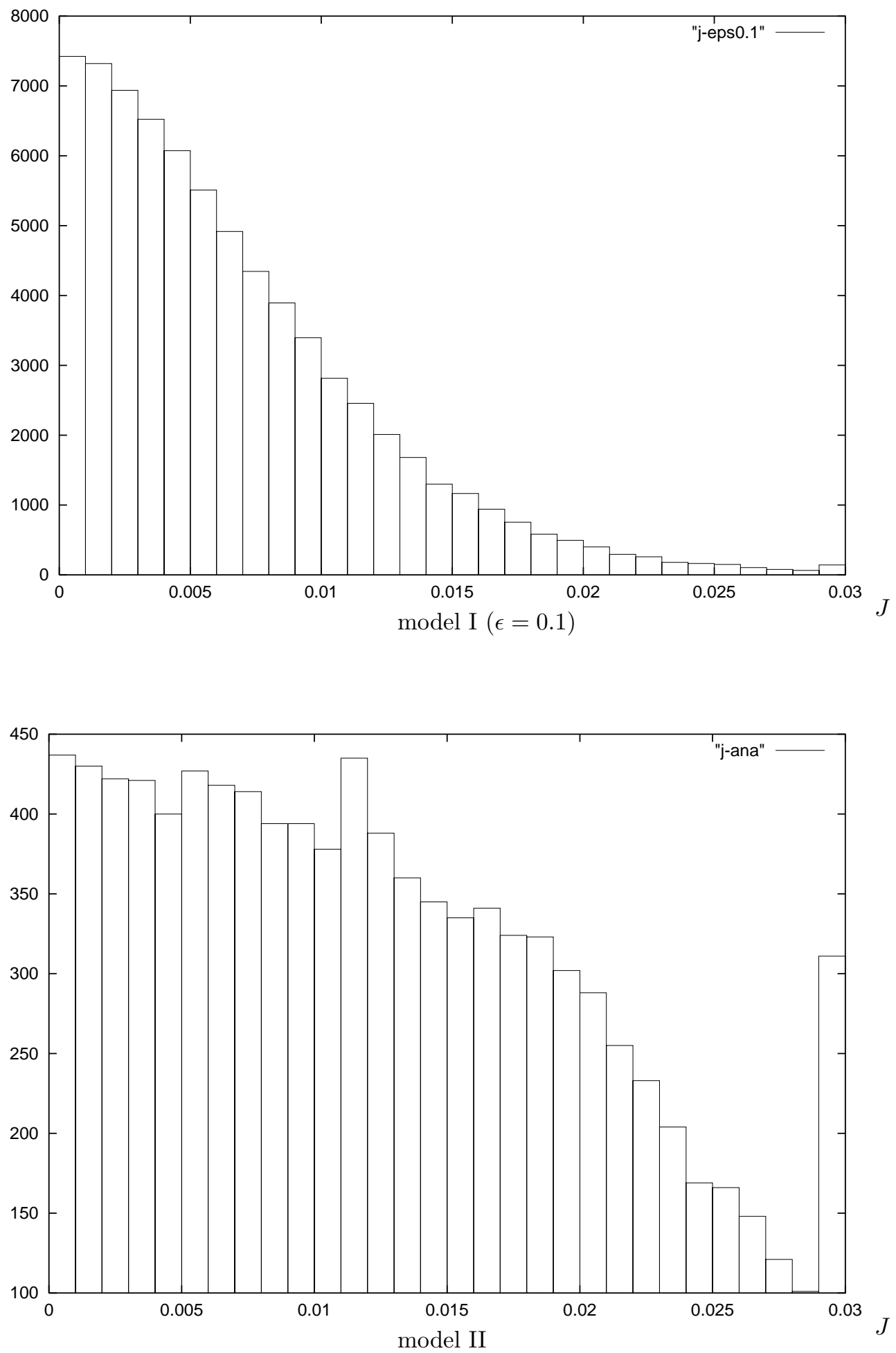

Figure 4: Distributions of $J$. The number of $J$ in the right bin means that of $J>0.029$. 

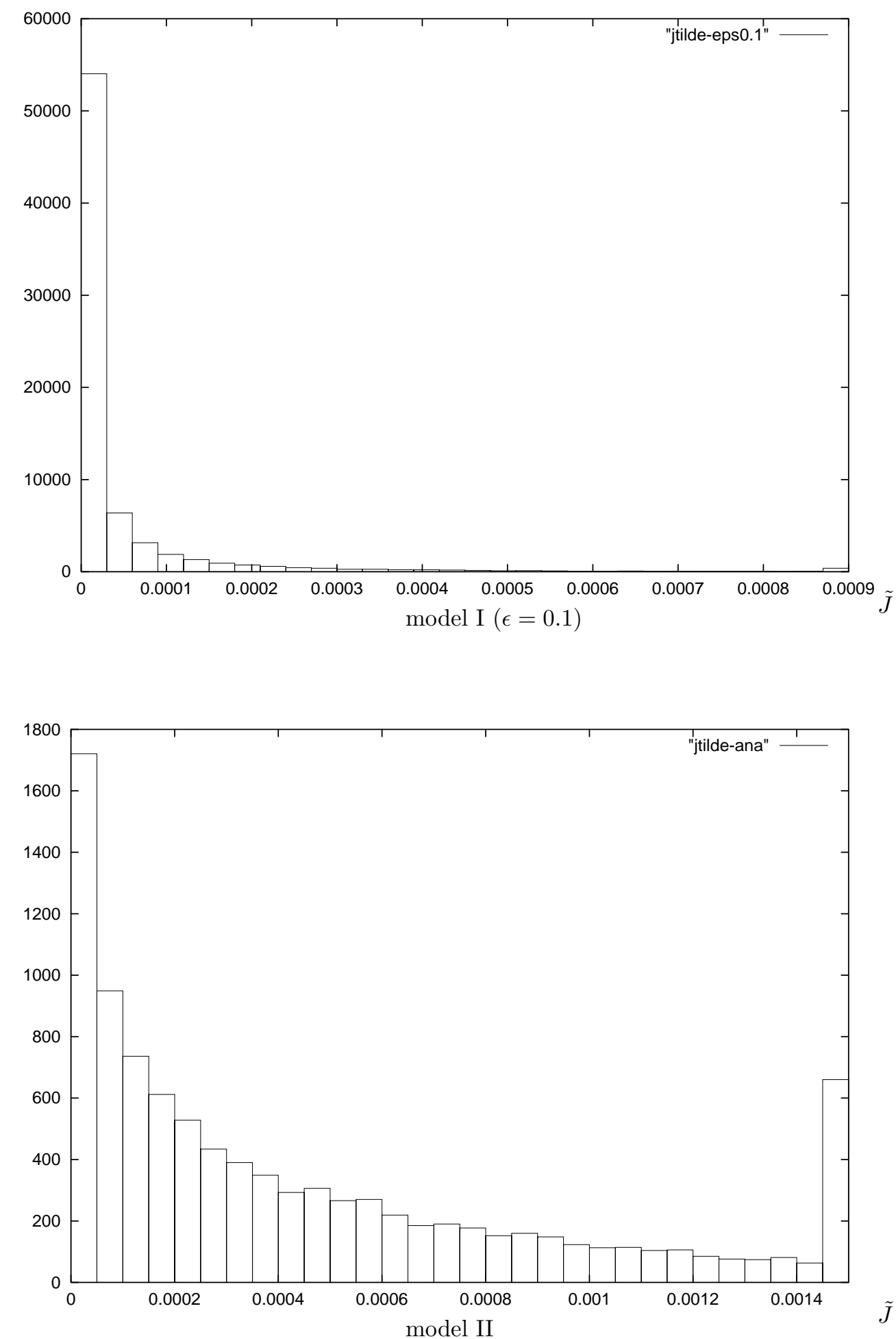

Figure 5: Distributions of $\tilde{J} \equiv|J \times r|$. The number of $\tilde{J}$ in the right bin means that of $\tilde{J}>0.0009$ for the model I and $\tilde{J}>0.00145$ for the model II. 

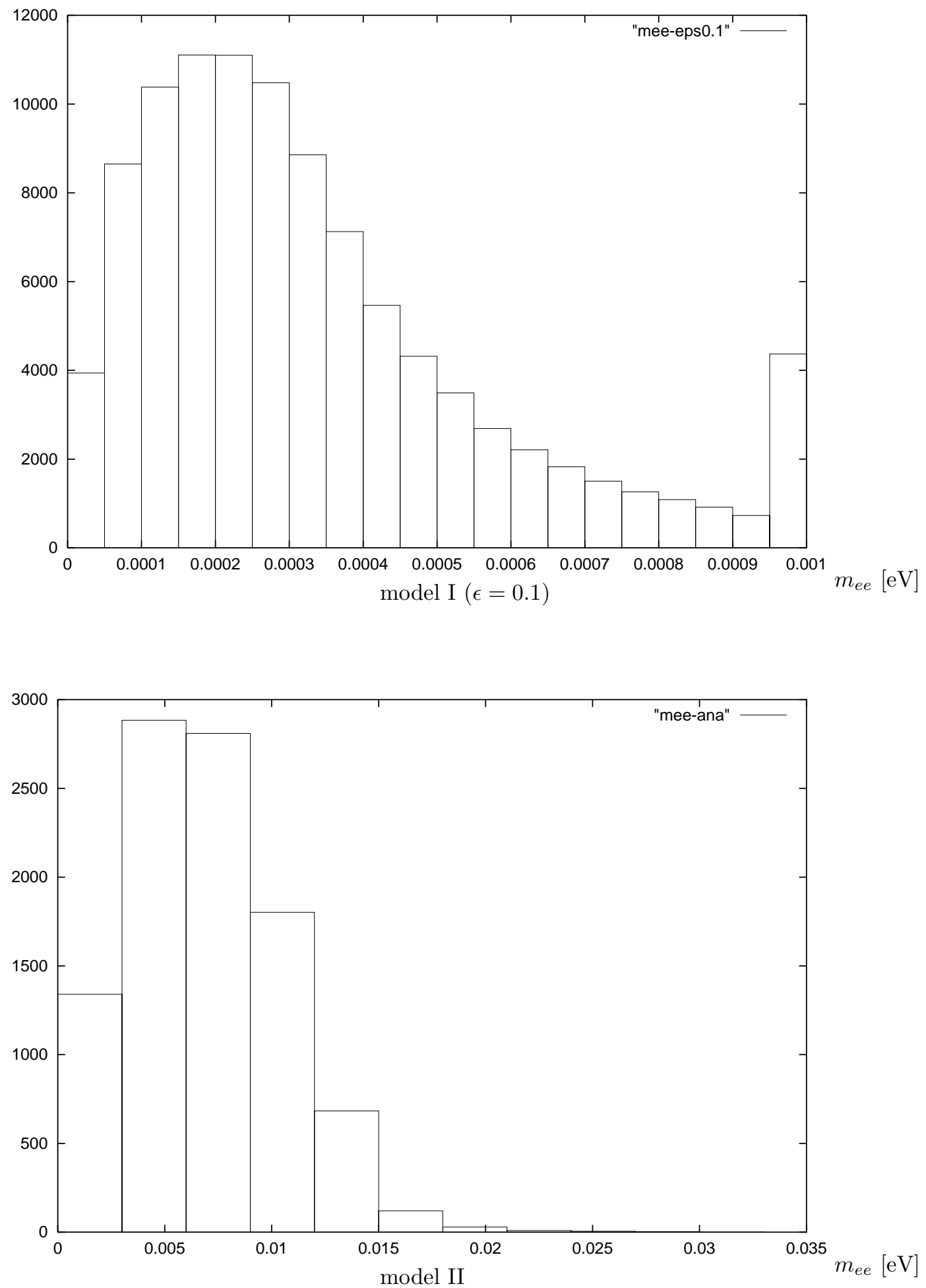

Figure 6: Distributions of $m_{e e}$. The number of $m_{e e}$ in the right bin for the model I means that of $m_{e e}>0.00095 \mathrm{eV}$. 